\title{
Kibreab, Gaim. - Eritrea. A Dream Deferred | Vincent, Léonard. - Les Érythréens. Récit
}

\section{Alain Gascon}

\section{(2) OpenEdition}

1 Journals

Édition électronique

URL : http://journals.openedition.org/etudesafricaines/14864

DOI : $10.4000 /$ etudesafricaines. 14864

ISSN : $1777-5353$

Éditeur

Éditions de l'EHESS

\section{Édition imprimée}

Date de publication : 22 novembre 2013

Pagination : 964-971

ISBN : 978-2-7132-2389-1

ISSN : 0008-0055

\section{Référence électronique}

Alain Gascon, «Kibreab, Gaim. - Eritrea. A Dream Deferred | Vincent, Léonard. - Les Érythréens. Récit », Cahiers d'études africaines [En ligne], 212 | 2013, mis en ligne le 06 décembre 2013, consulté le 24

septembre 2020. URL : http://journals.openedition.org/etudesafricaines/14864; DOI : https://doi.org/ 10.4000/etudesafricaines.14864

Ce document a été généré automatiquement le 24 septembre 2020.

(c) Cahiers d'Études africaines 


\title{
Kibreab, Gaim. - Eritrea. A Dream Deferred | Vincent, Léonard. - Les Érythréens. Récit
}

\author{
Alain Gascon
}

\section{RÉFÉRENCE}

KIBREAB, Gaim. - Eritrea. A Dream Deferred. Woodbridge, James Currey (« Eastern African Series »), Uppsala, Nordiska Afrikainstitutet, 2009, 448 p., bibl.

VINCENT, Léonard. - Les Érythréens. Récit. Paris, Éditions Payot \& Rivages (« Littérature »), 2012, 256 p.

1 Le titre du livre de Gaim Kibreab, professeur de la South Bank University de Londres, peut être rendu, par: Érythrée. Un rêve différé, reporté, ajourné, en sursis mais « un rêve devenu cauchemar " serait bien plus proche de son contenu. L'auteur, en effet, rédige un réquisitoire impitoyable et argumenté contre le régime du président, Isayyas Afäwärqi, assimilé aux pires dictateurs du XX siècle : Staline, Hitler, Mao et... Mängestu Haylä Maryam qui opprimal'Éthiopie et l'Érythrée de 1977 à 1991. L'acte d'accusation est dressé par l'auteur, un Érythréen qui a combattu trente ans pour l'indépendance de son pays. Son livre est plus qu'un pamphlet, c'est une étude minutieuse, parfois répétitive, mais toujours étayée par des documents, des ouvrages et des enquêtes auprès de plusieurs « centaines » de témoins. Concentrant tous les pouvoirs, Isayyas, sa « clique » et ses « suppôts » ont, en vingt ans, fait « main basse » sur l'Érythrée.

2 Le chef de l'État, du gouvernement et du Front populaire pour la justice et la démocratie (FPJD) ${ }^{1}$, le parti unique, est, également, président de l'Assemblée, chef des armées et président-fondateur du Hidri Trust Fund qui a absorbé toutes les entreprises privées, des plus grandes aux plus petites. Depuis le référendum de 1993, qui sanctionna la sécession d'avec l'Éthiopie et l'indépendance, aucune «vraie » élection 
n'a jamais eu lieu à aucun échelon que ce soit, et la constitution, ratifiée par l'Assemblée, n'a jamais été promulguée par le président (p.36). En dépit d'entorses répétées à la démocratie, d'ailleurs revendiquées par les autorités, cet État demeure membre de l'ONU et l'UA.

Le lecteur se demande à la suite de Gaim : «Comment en est-on arrivé là ? C Comment une insurrection "pure», une révolution «exemplaire», qui suscita tant de sympathies et d'espoirs en Afrique et dans le Tiers-Monde parmi les "progressistes », a dégénéré en un pouvoir absolu et solitaire. Dans sa préface : «Laver le linge sale en famille", il va au-devant des critiques qu'on n'a pas manquées de lui opposer. En "désespérant Asmära ", ne fait-il pas le jeu des ennemis de sa patrie, ne trahit-il pas trente ans de lutte populaire et ne se jette-t-il pas dans les bras de l'Éthiopie ennemie ? Le régime exerce un étroit contrôle sur les médias (censure, emprisonnement) et exhorte les Érythréens de la diaspora à garder le silence au nom de la solidarité nationale et les menaces d'arrêter leurs familles demeurées au pays. Remarquons, avec Gaim, que l'Érythrée, auréolée par sa longue lutte, a toujours bénéficié d'une extraordinaire complaisance de la part des prescripteurs d'opinion (médias, chancelleries, $\mathrm{ONG}$ ). En effet, en trente ans, une guérilla périphérique a rejoint les grands soulèvements populaires du Tiers-Monde à l'égal de la Palestine, du Vietnam et de Cuba. Alors que l'ONU et l'UA ont attendu la victoire des séparatistes pour reconnaître l'Érythrée, les réfugiés et les migrants des pays arabes ont très tôt réussi à populariser la cause du David érythréen qui luttait contre Haylä Sellasé, laquais du Goliath américain ${ }^{2}$. En dépit du ralliement de la junte éthiopienne (Därg) au marxismeléninisme en 1977, la cause n'en conserva pas moins son aura progressiste et gagna, en outre, l'appui du «monde libre ». Journalistes, militants, médecins sans frontières... se pressèrent alors dans les maquis du Sahel érythréen, exaltant la pugnacité des combattants et des combattantes, qui, unis dans la lutte, auraient aboli tous les clivages, de classe, de genre, de religion... Surpris et désorientés par le conflit éthioérythréen (1998-2000), qui opposa les deux alliés, vainqueurs de Mängestu, les commentateurs, en majorité, ont soutenu le David érythréen face au Goliath éthiopien. Or, cet affrontement n'était pas la continuation ou la reprise de celui de 1961-1991, mais une autre guerre comme bien des auteurs l'ont montré3. Mais l'impact des représentations sociales, fonds de commerce des médias, est plus fort que celui des travaux universitaires. Ainsi, ce n'est que dernièrement que la presse française, en retard par rapport aux journaux italiens notamment, a abandonné son attitude compréhensive vis-àvis de la dictature d'Asmära ${ }^{4}$.

On regrette que le livre ne présente aucune photographie ${ }^{5}$ et seulement trois cartes sommaires qui contrastent avec la pertinence des analyses de Gaim. Ce dernier a, en outre, décidé, à l'encontre de la règle adoptée par les éthiopisants et les "érythréisants ", de mentionner et classer les acteurs et les auteurs suivant l'usage européen alors que dans la vie courante celui qui, en Érythrée, parlerait d'Afäwärqi pour désigner le président Isayyas ne serait pas compris. En conséquence, dans cette recension, j'appellerai l'auteur Gaim, Gaim Kibreab mais jamais Kibreab! Sa transcription des termes en tigrinia (l'une des deux langues officielles d'Érythrée) est également très personnelle. Son propos se déroule en huit parties toujours étayées par de nombreux témoignages dont l'accumulation déroutera parfois le lecteur. Ces longs développements lui sont nécessaires pour lutter contre le courant d'opinion favorable, majoritaire, qui ressasse des représentations construites sur une histoire mythifiée et qui est soutenu par les moyens et les menaces de l'appareil de l'État érythréen. Gaim 
expose son réquisitoire en rappelant comment les autorités ont oublié les promesses de changements, utilisé la "guerre des frontières" avec l'Éthiopie (1998-2000) pour multiplier les violations des droits de l'homme et assujettir la vie associative. Il épingle le syndrome de la dépendance, le mythe de la souveraineté, l'obsession de ne compter que sur soi-même, slogans d'un pouvoir qui impose le monolithisme. Enfin, dans les derniers chapitres, l'auteur décrit comment le président a étouffé le secteur privé afin de mettre la main sur l'économie, interroge les divisions de l'opposition, reprend les promesses oubliées et s'attend à un retour de la famine. Tout au long de son livre, parfois difficile à suivre, il adopte un ton froid, contenu et solennel, mais qui accable le président et son régime, bien plus que la plus vengeresse des diatribes.

5 La taille et la complexité du livre montrent combien Gaim a peiné à faire le deuil de plus de trente années de lutte qui ont été au cœur de sa vie. En faire la recension n'est pas aisé car à chaque page, on trouve matière à réflexion, matière à citation. L'introduction mériterait, en elle-même, une longue discussion, notamment au sujet de l'avenir de l'Érythrée, colonie à laquelle l'Italie renonça en 1947, administrée de 1941 à 1951 par les Britanniques et enfin, fédérée à l'Éthiopie en 1952, à la suite d'un vote de l'Assemblée générale de l'ONU. Gaim rappelle qu'on a, tour à tour, projeté l'établissement d'un foyer national juif sur les hautes terres, la réunion des basses terres, en majorité musulmanes, de l'Ouest au Soudan et l'accès à la mer Rouge de l'Éthiopie à partir des plateaux, peuplés de chrétiens, du Centre, en échange de territoires en Ogadén. Il y eut, en Érythrée, des partisans de ce partage, d'un mandat italien, d'une union avec le Soudan, d'un retour à l'Éthiopie et enfin de l'indépendance immédiate. Il est dommage que l'auteur ne tienne pas compte des travaux qui montrent la part importante des indécis et leur versatilité et qui insistent sur les marchandages entre les grandes puissances et l'Éthiopie, membre fondateur de l'ONU ${ }^{6}$. On doit rappeler que la fédération a obtenu l'accord de la majorité des musulmans et des chrétiens et que la plupart des fonctionnaires et des militaires érythréens sont restés, jusque très tard, fidèles au gouvernement éthiopien ${ }^{7}$. En outre, entre 1952 et 1962, des partis érythréens ont aidé le gouvernement éthiopien à vider les institutions fédérales de leur contenu démocratique et à imposer l'annexion8.

6 Un climat de violence politique régnait déjà en Érythrée quand éclatèrent, dans les basses terres, les premières attaques des maquis. Ce long conflit, présenté comme une affaire de politique intérieure par le gouvernement éthiopien à l'ONU et àl'OUA, introduisit un clivage à l'intérieur de la société érythréenne jusque dans les familles d'abord comme une guerre civile qui, peu à peu, se mua en affrontement «national ». En effet, l'Érythrée dans ses frontières de 1897-1902, comprend, sur les plateaux centraux, des territoires dont les populations sont liées parl'histoire à l'Éthiopie et à ses périphéries des peuples demeurés hors de la sphère éthiopienne, mais rattachés par les hasards de la conquête coloniale ${ }^{9}$. Toutefois, le Front populaire de libération d'Érythrée (FPLE) qui est au pouvoir depuis 1991 à Asmära, est ancré sur les hautes terres du centre. Gaim rappelle (p. 37) que parmi le G15 (un groupe de 15 vétérans des luttes) qui rédigea, en 2001, un manifeste qui leur a valu la prison car il demandait au président la tenue d'élections, on trouve 11 membres de l'ex-Front de libération d'Érythrée (FLE). Ce front qui lança, dès 1960, la lutte dans les basses terres occidentales parmi les Beni Amer, des éleveurs musulmans, fut rejoint, dans les années 1970, par des étudiants marxistes d'Asmära (dont Isayyas). Ce groupe, qui avait séjourné en Chine (p. 225), opposé à l'idéologie arabo-musulmane du FLE, fonda le FPLE sur le modèle du FPLP, combattit les maquis du FLE (1973) et les expulsa d'Érythrée et des camps de réfugiés du 
Soudan (1981): «These fought a civil war against each other» (p. 319). La diaspora érythréenne dans les pays arabes ne peut rentrer au pays car les partisans du FLE y demeurent majoritaires ${ }^{10}$. L'indépendance n'a aucunement signifié la fin des hostilités et le pouvoir en place a utilisé cette "guerre civile froide " pour imposer sa complète hégémonie.

7 L'auteur (pp. 149-150) accuse, document à l'appui, le futur président d'avoir mené en 1973 une répression sanglante contre le FLE qui annonce les violences actuelles. Il instruit ainsi le procès du pouvoir en remettant en question le dogmede l'unité sans faille des Érythréens dans la lutte pour l'indépendance. Il ose affirmer : "One of the central arguments of this book is that Eritreans have always been governed by external rulers that recognised no brake and the outcome has been an unhappy one » (p. 27). En 1994, le $3^{e}$ congrès du FPLE (désormais FPJD) adopta la Charte nationale qui prévoyait la formation de people-based institutions (p.55) tandis que le gouvernement décidait de nommer les cadres des associations et des ONG. Ainsi, l'Eritrean Relief Association, qui avait ravitaillé les populations «libérées» pendant la guerre, perdit-elle toute autonomie et toute crédibilité auprès des donateurs. L'Université d'Asmära s'aligna sur le régime et l'Union nationale des paysans érythréens, instaurée en 1978, fut dissoute (p. 57). Les anciens combattants infirmes, lassés d'attendre la prime de démobilisation ayant manifesté à Asmära, se virent envoyer dans les campsde détention que les Éthiopiens avaient ouverts. Démobilisées en priorité, les combattantes ne reçurent guère de secours et durent réintégrer leurs villages où elles retrouvèrent leur condition d'avant la guerre. Quand, en 1998, éclata laguerre avec l'Éthiopie, l'ensemble de la vie associative était contrôlé par le parti unique et donc par le président. Plus qu'une "guerre des frontières " commel'écrit Gaim, ce fut un conflit d'État à État où chacun jeta toutes ses forces dans la bataille comme le montrent les pertes: 1 million de déplacés soit unquart des Érythréens (p. 133). Tout en manifestant son soutien à sa patrie, il assure que le commandement érythréen s'attendait à n'affronter que les maquis du Front populaire de libération du Tegray (FPLT), ses alliés contre Mängestu, alors qu'en Éthiopie, l'ensemble des peuples répondit à la mobilisation (p.31). Il reprend à son compte la déclaration d'un ancien chef d'état-major qui épingle une crise du leadership (p. 345).

8 Face au désarroi provoqué par la défaite, le G15, évoqué plus haut, rendit public, début 2001, un manifeste adressé au président lui demandant de réunir l'Assemblée, de promulguer la constitution et de la respecter. Isayyas reçut des délégations, promit et attendit six mois pendant lesquels sa propagande dénigra les opposants, les accusant de pactiser avec l'ennemi. Il déclara ensuite : "The EPLF has been an integral part of the blood and spirit of the people » (p. 4) et il est plus qu'un parti, un mouvement national dont le slogan est: «Un peuple, un cœur» (p. 187). Toute diversité de quelque ordre que ce soit est assimilée à une manipulation ou à une trahison : "The Eritrean identity is permanent, immutable - and non negociable " (Afwerki 2003) affirme le président (p. 206). Les carrières politiques se font et se défont au gré des coups de téléphone de la présidence qui ne notifie rien par écrit! Une lutte de tous les instants oppose ses proches qui lorgnent les faveurs matérielles afin de constituer des bases régionales où ils se retranchent face au pouvoir central. Parvenus à la tête des entreprises nationalisées, ces potentats ont bâti des fiefs locaux avec des prisonsprivées (pp. 275-276). L'État étant désormais le seul employeur, l'appartenance au FPJD est le sésame de tout emploi : il compte (en 2006) 600000 membres fichés et répertoriés (p. 5) soit un quart de la population adulte ${ }^{11}$. Le « nationalisme compulsif» (p. 388) prime sur 
tout autre souci et entraine les mesures les plus contradictoires et les plus discutables qui obèrent l'avenir et l'existence même de l'Érythrée. Le signe le plus évident de cette faillite est le «vote avec les pieds» (p. 291) des élites «bourgeoises» (p. 293) qui ne trouvent aucun emploi dans les entreprises nationalisées où règnent favoritisme et corruption. Les jeunes Érythréens forment, en effet, une part importante des migrants de la Corne de l'Afrique qui affrontent la mer Rouge ou le Sahara afin de gagner l'Europe. L'Université d'Asmära a été démantelée puis divisée (p. 89) en collèges régionaux où entrent les élèves, garçons et filles, des écoles secondaires après la classe de $11^{\mathrm{e}}$. Ils y subissent un dressage militaire et patriotique qui les prépare à un long service national, imposé de 18 à 50 ans, qui prive l'économie des cadres dont elle aurait besoin (p. 286). Ils sont employés, pour des salaires de misère, dans les firmes tombées sous la coupe des dirigeants du parti-État, dans les entreprises d'État : Hidri Trust Fund, Red Sea Trading Corporation. Le programme d'alphabétisation lancé dans les territoires libérés pendant la guerre d'indépendance (p.111) s'est peu à peu éteint. En 2002, alors qu'on négociait l'accord de paix avec l'Éthiopie, les dépenses de la défense atteignaient encore $23,5 \%$ du PIB, soit dix fois ce qui était alloué à l'éducation ou à la santé (p. 281). En 2006, l'armée comptait 300000 soldats (p. 186) alors que l'adversaire éthiopien, près de quinze fois plus peuplé, n'en enrôlait que $150000^{12}$.

Les pages consacrées à la caporalisation de l'économie sont particulièrement sinistres alors que l'Érythrée, même annexée à l'Éthiopie, possédait des entreprises florissantes qui bénéficiaient du marché éthiopien (bières Melotti, allumettes, textiles Barotollo, agro-industrie De Naddai) ${ }^{13}$. «Prior to Eritrea's annexation into Ethiopia in September 1962, there was a relatively well-functionning private sector » (p. 226). Les destructions de la guerre et les nationalisations décrétées par la junte éthiopienne (Därg), n'avaient pas fait disparaitre l'industrie : en 1991, il y avait 42 usines nationalisées et 650 petites entreprises privées (p. 226). Après la reprise en main de 2001, la situation de l'appareil de production s'est dégradée. Enfermés dans la «mentalité du bunker» (p.122), le président et ses acolytes ont stigmatisé l'aide étrangère qui introduit l'esprit de paresse et de corruption chez les Érythréens. Elle a pourtant nourri, de 1985 à 1995, entre 50 \% et $80 \%$ de la population et l'a aidée à passer le cap de la guerre éthio-érythréenne. La diminution brutale de l'aide alimentaire internationale (de 1,3 million à 72000 bénéficiaires !) décrétée en septembre 2005, s'est traduite par le doublement des cas de malnutrition (p. 155). Pour desserrer l'étau de la misère, les autorités ont ressuscité les échoppes de quartier et rétabli les achats franco valuta (p. 254) ${ }^{14}$ comme le fit le Därg, encourageant ainsi la contrebande et le trafic de devises. L'auteur signale que les uqub, les associations traditionnelles d'épargne volontaire avec tirage au sort, sont le seul espoir d'une population paupérisée (p.311). Notons enfin que le gouvernement ne publie pas de budget régulier (p.335), exige des impôts rétroactifs (p. 289), ne promulgue pas le code du travail (p.371) et astreint la population à un passeport intérieur (p. 365).

Il faut arrêter là l'énumération des épreuves vécues par les Érythréens qui vident l'Érythrée de sa jeunesse et de ses élites, contraintes de quitter leur pays de peur d'être emprisonnées sans jugement et sans aucune communication avec l'extérieur. Gaim Kibreab nous donne, heureusement, une liste de ces détenus oubliés, mais aussi les noms des 28 diplomates qui ont fait défection pour l'exil (p. 318). Les nombreux tableaux statistiques appuient le caractère implacable du réquisitoire comme les entreprises intégrées dans le Hidri Trust Fund. Ce livre fournit des renseignements d'une grande précision sur la constellation des mouvements érythréens, pour la 
plupart en exil et déchirés par des rivalités inexpiables. Il s'interroge, et nous interroge également, sur l'extraordinaire mansuétude dont le David érythréen, archétype du révolté du Tiers-Monde, a bénéficié (et bénéficie) de la part des médias, des humanitaires, des militants et... de certains chercheurs. Gaim, pour expliquer les " dérives » violentes et autoritaires du régime, renvoie, à juste titre, à la guerre civile qui opposa les deux fronts et qu'on a cherché à oublier. Il doit remonter à la période de la fédération où la lutte pour l'indépendance ne faisait pas l'unanimité parmi les partis érythréens, prompts à se tourner vers le bras armé éthiopien, pour éliminer un adversaire. Il faudrait aussi éclaircir les débuts du FLE au Caire, où siégeait, parmi les musulmans, un chrétien Wäldä $\mathrm{Ab}$ Wäldä Maryam. Les fondateurs du front y rencontrèrent les militants en exil du FLN algérien, copièrent ses structures et s'inspirèrent, sans doute, de son intransigeance. La défaite du FLE ne rappelle-t-elle pas l'échec du MNA, le rival du FLN, qui fut chassé des maquis algériens et des bidonvilles de France comme le FLE le fut d'Érythrée et des camps du Soudan?

11 Le livre de Gaim Kibreab, qui lui a demandé un extraordinaire effort de distanciation par rapport à sa vie militante, fait date dans la bibliographie consacrée à l'Érythrée. Nul ne peut ignorer cet ouvrage ou jamais la rigueur, l'honnêteté et la lucidité de l'auteur ne sont prises en défaut.

12 Le « récit » de L. Vincent, comme le livre de Gaim Kibreab, dresse un réquisitoire contre le régime d'Isayyas Afäwärqi, mais de l'« extérieur». Jamais, et c'est sans doute heureux pour sa liberté personnelle, ce journaliste n'a pu entrer en Érythrée où ses confrères sont en fuite ou en prison. Il a recueilli ses informations lors de rencontres avec de nombreux réfugiés érythréens à Khartoum, en Éthiopie, dans les pays arabes, en Europe et aux États-Unis. Il retrace ainsi le parcours, semé d'embûches, de ceux, peu nombreux, qui ont réussi à franchir les frontières en dépit des rafles, des contrôles, des arrestations, des emprisonnements, des tortures, des gardes, des mines et de l'avidité des passeurs. Tous les témoignages rapportés sont poignants et parfois leur mise en scène est par trop spectaculaire. Même si son expression manque parfois de sobriété, l'auteur a recoupé et authentifié les récits qui corroborent, en tous points, les analyses de Gaim. Après avoir subi trente ans de guerre, depuis vingt ans, les Érythréens vivent sous la dictature. En dépit d'inexactitudes et d'approximations ${ }^{15}$, fruits de lectures hâtives, L. Vincent fait, justement, remonter les racines de cette dictature, non pas à la dérive ou dans la réaction d'un pouvoir menacé, mais dans les racines mêmes de la lutte pour l'indépendance. Ceux qui ont pris le pouvoir à Asmära en 1991 ont éliminé, dans les maquis et les camps de réfugiés, ceux qui se sont soulevés en 1961. La guerre d'indépendance érythréenne fut une guerre civile, plus âpre que ne l'écrit Gaim. L. Vincent apporte une pierre nouvelle au débat sur le black-out qui entoure le sort des Érythréens qui fuient la dictature. Il nous relate comment, à Rome, les «moustiques » de l'ambassade d'Érythrée ont réussi à prendre le contrôle d'un immeuble où se regroupaient les réfugiés. Il est, toutefois, un peu court dans ses analyses géopolitiques et charge la barque d'Isayyas, sorte de génie du mal totalitaire. Or, comme Gaim le précise et comme les historiens l'ont démontré, un pouvoir solitaire a besoin de relais pour s'imposer par la fidélité, la terreur, l'appât du gain et des honneurs...

13 Pour les lecteurs francophones qui ne savent rien d'autres de l'Érythrée que les clichés remontant à la saga de l'indépendance, le récit de L. Vincent est une bonne mise au point sur la Corée du Nord africaine et c'est surtout une invitation à lire Gaim Kibreab. 


\section{NOTES}

1. En anglais : People's Front for Democracy and Justice (PFDJ).

2. Hélène THIOLLET, Migrations et intégrations dans le Sud de la mer Rouge. Migrants et réfugiés érythréens au Yémen, au Soudan et en Arabie Saoudite 1993-2007, Thèse de doctorat de science politique, Paris, IEP, 2007.

3. Tekeste Negash \& Kjetil TRONVoll, Brothers at War. Making Sense of the Eritrean-Ethiopian War, Oxford, James Currey ; Athens, Ohio University Press, 2000. Pour la guerre de 1961-1991, voir Paul B. HENZE, Ethiopia in Mengistu's Final Years. Until the Last Bullet, vol. 2, Addis Ababa, Shama Books, 2007, et surtout Marc FonTRIER, La chute de la junte militaire éthiopienne (1987-1991), Paris, L'Harmattan-Aresæ (« Bibliothèque Peiresc, $13 »), 1999$.

4. Alexandra GENESTE, «L'ONU envisage de nouvelles sanctions contre l'Érythrée », Le Monde, 6 décembre 2011. François SOUDAN, «Érythrée : Bienvenue dans la Corée du Nord de l'Afrique », Jeune Afrique, 50 année, $\mathrm{n}^{\circ} 2$ 565, 7-13 mars 2010.

5. Le lecteur se référera à Pount, 4, 2010 : Anna Godıo, «Assab: genesi e crescita di una città dimenticata ", pp. 9-25; Marie BRIDONNEAU, "Patrimonialiser le centre colonial d'Asmara », pp. 27-51; David BozzinI \& Roberta DEAmBrosi, «Asmara ou les logiques de la contrainte», pp. 53-69.

6. Rossi GiANLUIGI, L'Africa italiana verso l'indipendenza (1941-1949), Rome, Giuffrè editore, 1980. Tekeste NEGASH, Eritrea and Ethiopia. The Federal Experience, New Brunswick, Transaction Publishers, 2005.

7. Tafla BAIRU, « Eritrea in Retrospect. An Excerpt from the Memoirs of Fitawrari Mika'el Hasama Rakka », Africa (Roma), LX (1), Marzo 2005, pp. 1-64.

8. TEKESTE, op. cit.; Medhanie Tesfatsion, Eritrea. Dynamics of a National Question, Amsterdam, B. R. Grüner, 1986.

9. Le roman de Luca L UCARELli, La huitième vibration, Paris, Métailié, 2010, le montre excellemment.

10. H. THIOLLET, op. cit.

11. Population \& Sociétés ( $n^{\circ} 480$, juillet-août 2011), dénombre 5,9 millions d'Érythréens dont 3,36 millions (57\%) ont plus de 15 ans.

12. Thèse de Patrick FERRAS soutenue à l'IFG (Paris 8) le 4 mai 2011.

13. Témoignage personnel (1970-1972).

14. On peut importer les marchandises avec des droits de douane réduits pourvu qu'on paye comptant en devises. Personne ne demande l'origine des devises.

15. Qu'on peut corriger en consultant l'Encyclopaedia Aethiopica, op. cit. 